\title{
Multiscale Modeling of Grain-Boundary Fracture: Cohesive Zone Models Parameterized from Atomistic Simulations
}

\author{
Edward H. Glaessgen ${ }^{*}$ and Erik Saether ${ }^{\dagger}$ \\ NASA Langley Research Center, Hampton, Virginia, 23681 \\ Dawn R. Phillips \\ Lockheed Martin Space Operations, Hampton, Virginia, 23681 \\ and \\ Vesselin Yamakov \\ National Institute of Aerospace, Hampton, Virginia, 23666
}

\begin{abstract}
A multiscale modeling strategy is developed to study grain boundary fracture in polycrystalline aluminum. Atomistic simulation is used to model fundamental nanoscale deformation and fracture mechanisms and to develop a constitutive relationship for separation along a grain boundary interface. The nanoscale constitutive relationship is then parameterized within a cohesive zone model to represent variations in grain boundary properties. These variations arise from the presence of vacancies, intersticies, and other defects in addition to deviations in grain boundary angle from the baseline configuration considered in the molecular dynamics simulation. The parameterized cohesive zone models are then used to model grain boundaries within finite element analyses of aluminum polycrystals.
\end{abstract}

\section{Introduction}

$\mathrm{P}$ HYSICS-based modeling of fracture begins at nanometer dimensional scales in which atomistic simulation is used to predict the formation, propagation, and interaction of fundamental damage mechanisms. These mechanisms include dislocation formation and interaction, interstitial void formation, and atomic motion such as Coble creep. The development of these damage mechanisms progress into microscale processes such as local plasticity and small crack formation. Ultimately, damage progression leads to macroscopic failure modes such as plastic yielding of components and large cracks exhibiting Mode I, II, and III opening behavior. Thus, multiscale modeling strategies are needed for the purpose of developing a unified description of the hierarchy of processes that govern fracture.

One strategy for multiscale modeling is to map an atomistic molecular-dynamics (MD) simulation of physical behavior at the nanoscale into continuum finite element representations for microscale analyses. These damage mechanisms can include the physical processes that govern intergranular and transgranular damage initiation and propagation in metallic microstructures and may be used to define effective traction-displacement relationships for modeling fracture at larger length scales. Once the traction-displacement relationships from MD are known, they can be used to define effective cohesive zone elements that can be placed along all possible fracture interfaces in a finite element mesh. Finite element simulations can then be used to study the failure properties of the material at larger scales. The methodology is very generic and can be used for any interface that is subjected to fracture provided that the atomistic simulation captures all relevant mechanisms. However, because of limitations on the domain size in the molecular-dynamics simulation (with typical dimensions on the order of tens of nanometers), this direct approach may be limited to simulating microstructures with characteristic dimensions on the order of microns.

\footnotetext{
${ }^{*}$ Aerospace Engineer, Computational Structures and Materials Branch, MS 155, AIAA Associate Fellow.

${ }^{\dagger}$ Aerospace Engineer, Computational Structures and Materials Branch, MS 155, AIAA Member.

* Aerospace Engineer, MS 155, AIAA Member.

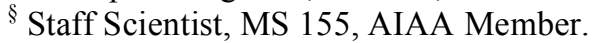


Several examples include fracture along grain boundaries in metals (intergranular fracture), fracture along weak slip planes (transgranular fracture), and fracture through interfaces between grains and small second phase particles.

The key to this approach is the recasting of the atomistic results within the framework of a continuum cohesive zone model. Cohesive zone models (CZMs) approximate traction-displacement relationships along a material interface and are frequently used in conjunction with the finite element method (FEM). They are embedded into zero-thickness CZM elements, which may be placed between continuum finite elements to simulate interface failure. In fracture studies at the micromechanics level, CZM elements can be used to predict transgranular fracture if they are placed adaptively between continuum finite elements within grains or to predict intergranular fracture if they are placed along grain boundaries (GBs) as shown in Figure 1.

Parameterizing the relative influence of various atomistic mechanisms and crystallographic orientations is important if CZMs are to be used effectively in a multiscale modeling strategy. In the present study, molecular dynamics (MD) simulations based on a bicrystal model are used to extract the combined elastic and plastic properties of a common grain boundary in aluminum under Mode I opening. The information is then recast in the form of bilinear CZMs to represent the mechanisms involved in fracture. This basic functional form is then used parametrically to study the effect of CZM parameters (representing variations in properties of individual grain boundaries) on grain boundary fracture within aluminum polycrystals.

In this paper, multiscale modeling is developed to study intergranular fracture in aluminum with cohesive zone models implemented to bridge length scales. First, the basic formulation, numerical features and parameterization of the CZMs based on MD simulation are discussed. Second, the MD analysis, including the extracted mechanisms of atomic-scale deformation and fracture, is reviewed. Third, the method for parameterizing the tractiondisplacement response along the fracturing grain boundary is presented. Finally, sample results based on this parameterization for both a bicrystal and a polycrystal are discussed.

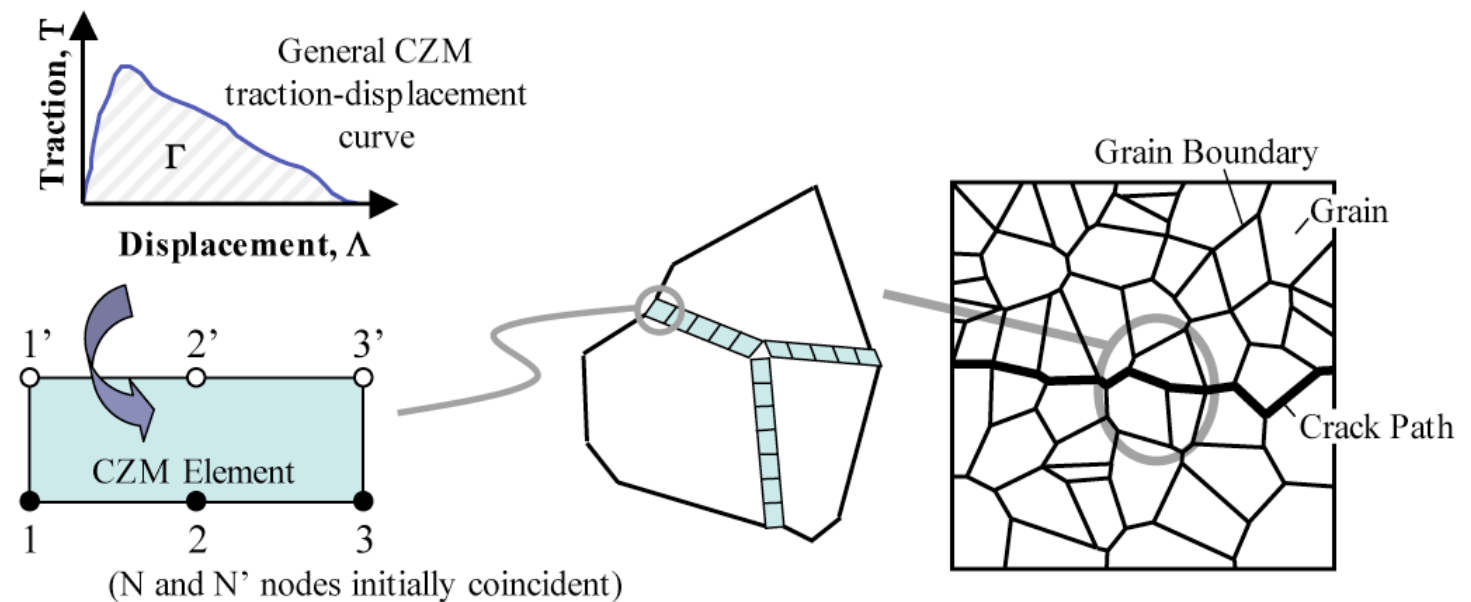

Figure 1: Embedding CZMs along GBs to study microstructural fracture.

\section{Finite Elements Incorporating Cohesive Zone Models}

\section{A. Cohesive Zone Models}

Numerous CZM formulations have been presented in the literature (Needleman, 1990, Tvergaard and Hutchinson, 1992; Costanzo and Allen, 1995; Camacho and Ortiz, 1996; Klein and Gao, 1998; Turon, et al. 2004). A general review of various forms of CZM is found in Chandra, et al. 2002. Central to most formulations is the requirement that the area under the CZM traction-displacement curve represents the work of separation required to open a crack or the fracture toughness and is given by Tvergaard and Hutchinson (1992) as

$$
G_{c}=\int_{0}^{\Delta^{f}} \tau d \Delta
$$


where $G_{c}$ is the work of separation, $\tau$ is a general form of the traction, $\Delta$ is a general form of the displacement, and $\Delta^{f}$ is the critical displacement at which complete separation has occurred and the tractions are zero.

A mixed-mode CZM developed by Turon et al. (2004) has been used in the current investigation wherein the normal and shear components of the traction and displacement are interpolated based on mode mixity. A brief outline of the basic formulation given by Turon et al. (2004) is presented here. The interpolation of CZM components is depicted in Figure 2. An interpolated CZM assumed in a bilinear form in which relative displacements between the upper and lower nodes, $\Delta=\delta^{\text {top }}-\delta^{b o t}$, indicate the onset of damage, and final failure of the cohesive zone is shown in Figure 3.

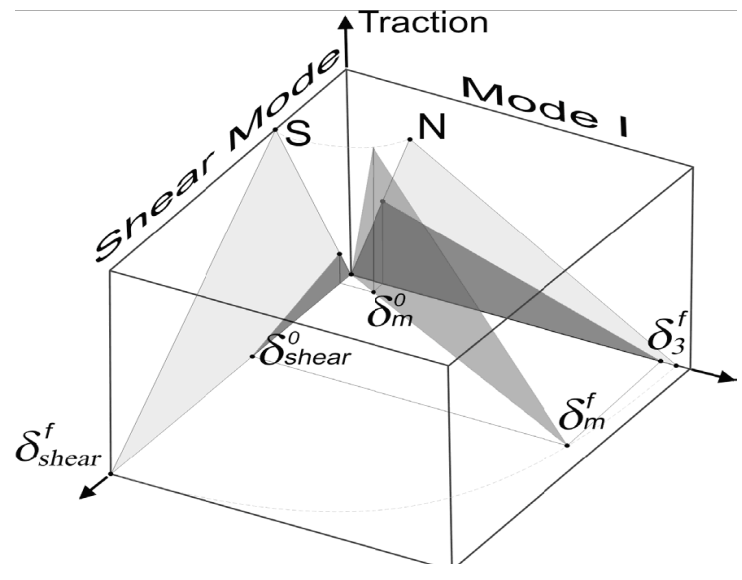

Figure 2. Interpolation of CZM components. (From Turon et al 2004.)

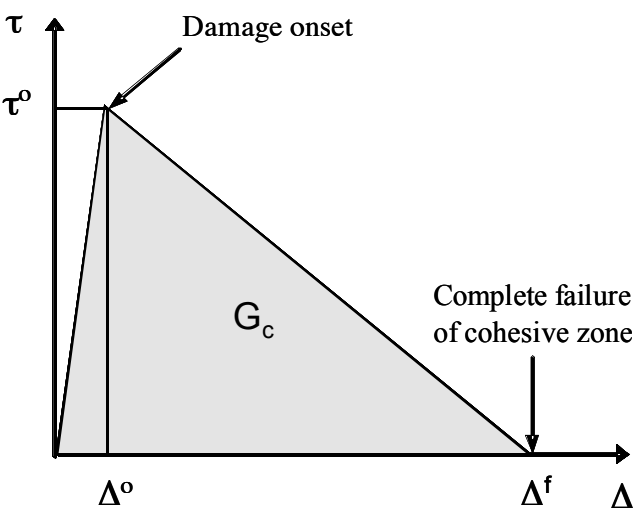

Figure 3. Bilinear cohesive zone model.

An equivalent relative displacement is defined as

$$
\lambda=\sqrt{\left\langle\Delta_{3}\right\rangle^{2}+\Delta_{\text {shear }}^{2}}
$$

where the Macaulay bracket is defined as $\left\langle x>=1 / 2(x+|x|), \Delta_{3}\right.$ is the non-negative relative displacement in Mode $\mathrm{I}$, and $\Delta_{\text {shear }}$ is the combined relative displacement in Mode II and Mode III given by

$$
\Delta_{\text {shear }}=\sqrt{\left(\Delta_{1}\right)^{2}+\left(\Delta_{2}\right)^{2}}
$$

The mode mixity ratio is defined as

$$
\beta=\frac{\Delta_{\text {shear }}}{\Delta_{\text {shear }}+\left\langle\Delta_{3}\right\rangle}
$$

The effect of combining individual fracture modes on fracture evolution is characterized by assumed interactions of the various fracture toughness measures, $G$. The strain energy release rate, $G$, is computed by relating the critical fracture toughness measures for Mode I, II, and III given by $G_{I c}, G_{I I c}$, and $G_{I I I c}$, respectively, together with the current measures for the strain energy release in shear, $G_{\text {shear }}$, and the total strain energy release rate, $G_{\text {tot }}$. An effective measure for $G$ is given by the model of Benzeggagh and Kenane (1996). This model is utilized in the present study and is given by

$$
G=G_{I c}+\left(G_{I I c}-G_{I c}\right)\left(\frac{G_{\text {shear }}}{G_{\text {tot }}}\right)^{\eta}
$$


where $G=G_{I}+G_{\text {shear }}$ and $G_{\text {shear }}=G_{I I}+G_{I I I}$. The ratio of the combined shear and the total strain energy release rate may be expressed in terms of the mode mixity ratio as

$$
B=\frac{G_{\text {shear }}}{G_{\text {tot }}}=\frac{\beta^{2}}{1+2 \beta^{2}-2 \beta}
$$

The mixed-mode damage onset displacement is calculated as

$$
\Delta^{o}=\sqrt{\left(\Delta_{3}^{o}\right)^{p}+\left[\left(\Delta_{\text {shear }}^{o}\right)^{p}-\left(\Delta_{3}^{o}\right)^{p}\right][B]}
$$

and the mixed mode displacement corresponding to complete failure of the cohesive zone is given by

$$
\Delta^{f}=\frac{2}{K \Delta^{o}}\left[G_{I c}+\left(G_{I I C}-G_{I c}\right)[B]_{-}^{-}\right.
$$

Assuming a bilinear CZM as shown in Figure 3, a damage parameter, $d$, is computed as

$$
d=\frac{\Delta^{f}\left(\lambda-\Delta^{o}\right)}{\lambda\left(\Delta^{f}-\Delta^{o}\right)}
$$

The resulting constitutive law is given by

$$
\tau=\left\{\begin{array}{c}
\tau_{1} \\
\tau_{2} \\
\tau_{3}
\end{array}\right\}=(1-d) k\left\{\begin{array}{c}
\Delta_{1} \\
\Delta_{2} \\
\Delta_{3}
\end{array}\right\}-d k\left\{\begin{array}{c}
0 \\
0 \\
\left\langle\Delta_{3}\right\rangle
\end{array}\right\}
$$

For the bilinear CZM presented in Turon et al. (2004), $k$ is the initial slope of the traction - displacement law, which in this case when the GB width is non-negligible, represents the GB stiffness.

\section{B. Parameterizing Cohesive Zone Models}

While CZMs are a useful method to simulate material failure, because the parameters that are typically used to define them are taken from bulk material properties (Iesulauro, 2002), they do not describe adequately the actual physics of microstructural fracture. Macroscale values of strength and toughness that are typically input to the CZM represent the aggregate responses of thousands or millions of grains, grain boundaries, and defects within the specimens from which they were obtained. Thus, these macroscale values do not represent the unique response of a particular interface at which a local fracture event might occur. If the microscale predictions are to become quantitative, consideration of the local nanoscale properties is required. One possible means of making this connection is to use the results of MD models as input to the CZM. This connection would allow more realistic simulations leading to accurate predictions of the failure properties of a large class of materials and microstructures, even when experimental data is not available.

Attempts to extract relevant parameters for the decohesion law of a CZM from atomistic (molecular-dynamics, molecular-static, or 'first-principle') simulations have been made by various groups in the last few years (Gall et al., 2000; Komanduri et al., 2001; Spearot et al., 2004; Raynolds et al., 1996). The approach in all of these works is based on simulating the debonding of a flat interface under a constant tensile strain rate perpendicular to the interface. In these references, the system size is between 4 and $8 \mathrm{~nm}$, and the dynamics of the atoms is severely constrained by the boundary conditions, which do not allow for Poisson lateral contraction and shear deformation. As a result, plastic processes, such as dislocation slip, are strongly suppressed. Consequently, the simulated mechanism for interface decohesion in these references reproduces the process of atomic adhesion (strength) rather than that of fracture at the interface. 
The boundary conditions at which a typical CZM element operates in a large-scale finite element (FE) model are very different from the ones used in the referenced MD and first principles simulations. Typically, the CZM elements are embedded within a system of finite elements that reproduce the elastic and plastic response of the surrounding material to both the external load and the crack-tip stress. In contrast, decohesion parameters, such as peak stress and opening displacement of the CZM curve, are typically extracted from an atomistic volume of order $10 \mathrm{~nm}$ in each direction in the referenced simulations. The lack of an adequate surrounding volume of material and the stiff boundary conditions suppresses the plastic processes, such as dislocation nucleation, limiting the accommodation of deformation at the interface and forcing the configuration to debond in an unnatural manner. The periodic boundary conditions usually applied in these models cause the simulation to create a response of an array of repeating units with a strong overlap of image elastic forces rather than the response of a single specimen unit. Consequently, the resulting decohesion curves cannot be directly applied to derive the constitutive laws for CZM elements.

Thus, an MD-based CZM decohesion law for intergranular fracture under local conditions similar to those experienced by the CZM element in a polycrystalline FE model is developed in the present work. The CZM decohesion law reflects the response of the CZM element to an approaching and propagating crack (Costanzo and Allen, 1995; Dávila, 2001). The present MD model is a model of crack propagation rather than of adhesion and is used to simulate a crack propagating through a flat high-energy grain-boundary in aluminum (Yamakov et al., 2005).

\section{Development of a Cohesive Zone Model Based on MD Simulation}

\section{A. Configuration Considered in Molecular Dynamics of Grain Boundary Separation}

For metallic microstructures, the effective GB thickness is small. Figure 5 shows a typical $\Sigma 99$ GB formed between two grains in an fcc metal. Common neighbor analysis (CNA) (Honeycutt and Andersen, 1987; Clarke and Jonsson, 1993) is used to identify atoms in different crystallographic states: fcc (small dots), hep (triangles), and non-crystalline atoms (large dots). Atoms with more than $1 / 3$ of their nearest neighbors missing are identified as surface atoms (squares), indicating existing vacancies in the GB. The length scale is in units of the lattice constant of Al, $a_{o}=0.405 \mathrm{~nm}$ (Mishin et al., 1999). Here, the specific problem of intergranular fracture is considered wherein MD simulations of fracture along GBs are used to obtain the atomistic response of crack propagation under applied loads along a characteristic length of the GB.

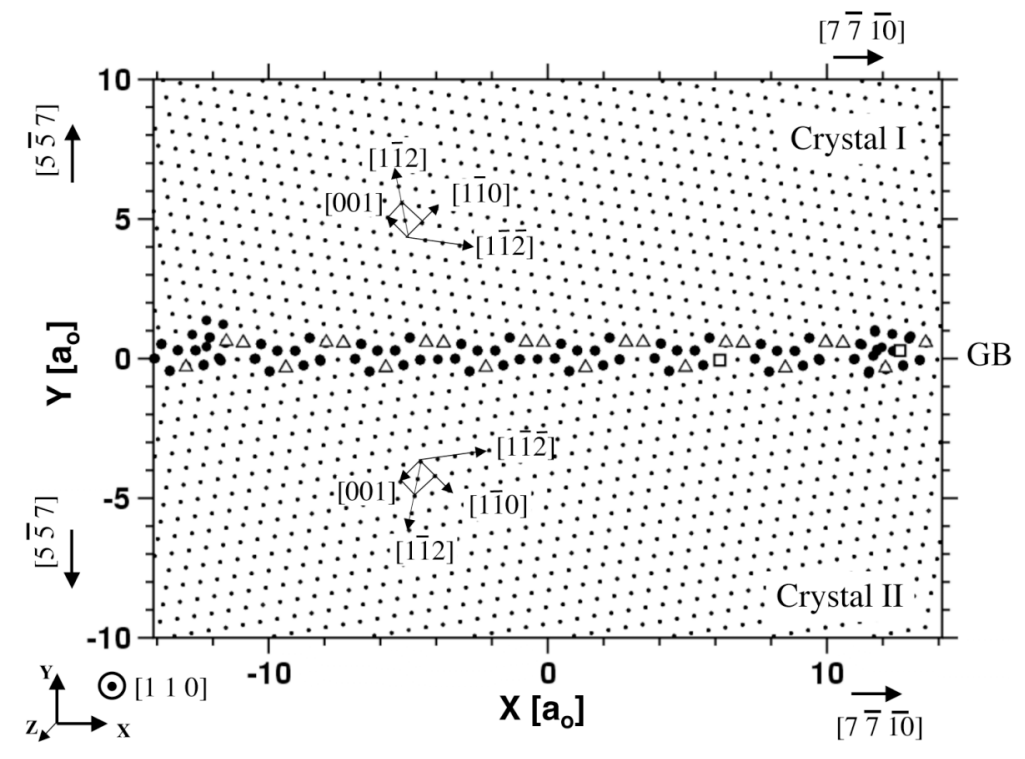

Figure 5. Atomistic snapshot giving the crystallography and structure of the GB interface.

\section{B. Plastic Contribution to the Stress Field Near the Crack}

The results of a molecular dynamics simulation developed by Yamakov and coworkers (Yamakov et al., 2006) that reveals many details of the crack propagation and dislocation response in the bicrystal system are summarized here. Figure 6 shows the deformation mechanisms and crack growth of the atomic system resulting from four 
different initial hydrostatic stresses: $\sigma=3.5$ (a), 3.75 (b), 4.0 (c), and 4.25 GPa (d). CNA is used to identify atoms in different crystallographic states: fcc (in gray), hcp (in red), non-crystalline atoms (in blue), and surface atoms (in green). Thus, a number of different formations are indicated in the figure as follows: (1) - GB interface; (2) - twin boundary; (3) - core of a partial or twinning dislocation; (4) - nanovoid at the crack tip; (5) - slip dislocation; (6) - GB dislocation; and 7 - secondary slip. Due to the crystallographic orientation of the grains, the crack induced along the grain boundary propagates dissimilarly in the $+x$ and $-x$ directions. The crack tip is blunted in the $-x$ direction as is characteristic of ductile fracture whereas the crack tip remains sharp in the $+x$ direction as is characteristic of brittle fracture. The deformation in the $-x$ direction is dominated by the creation of a series of nearly symmetrical twins. Although twinning is not common in Al, the preference for twinning to dislocation slip is in agreement with the Tadmor and Hai criterion (Tadmor and Hai, 2003).

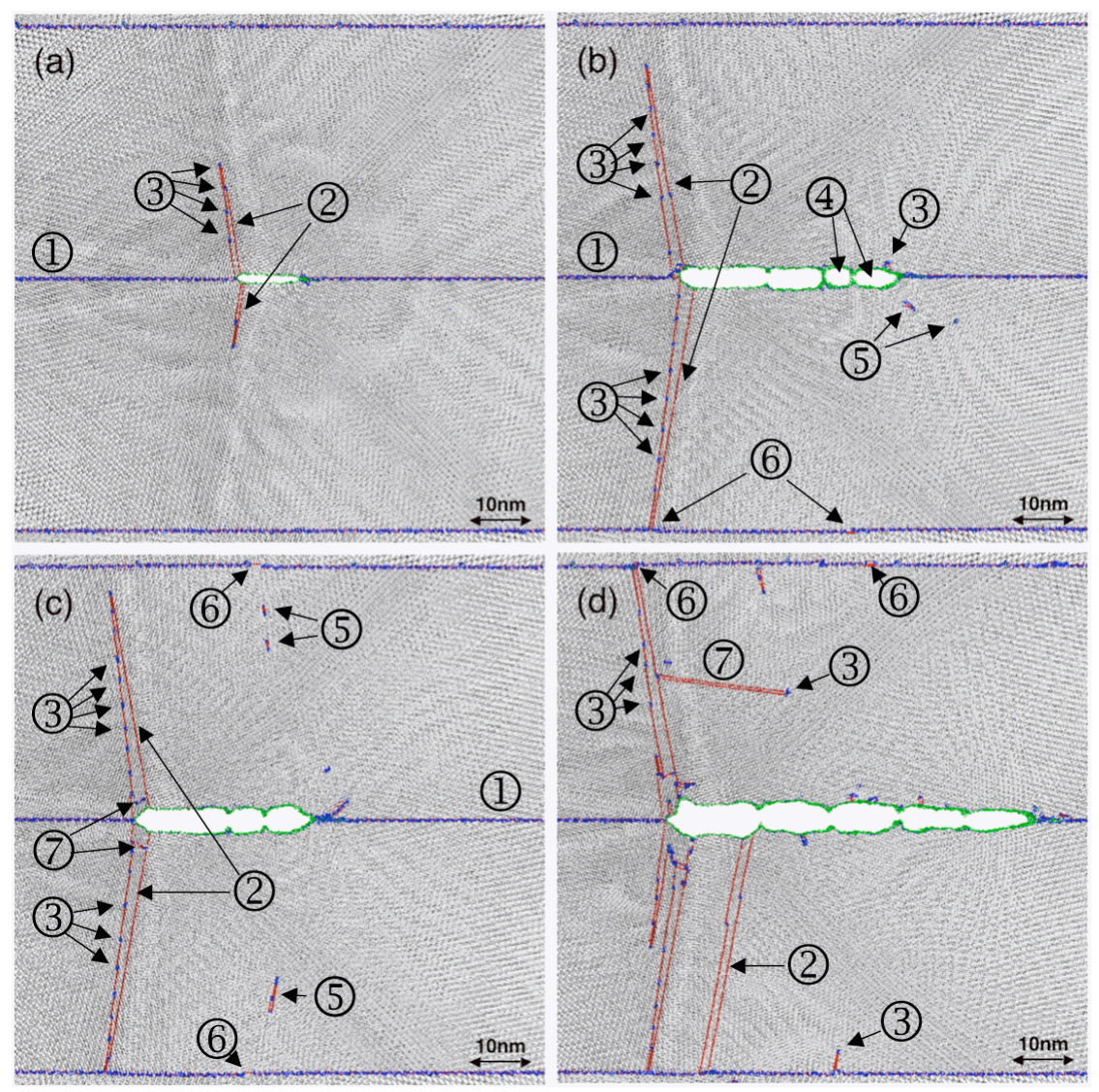

Figure 6. Snapshots of cracks that have propagated in the MD system.

\section{Development of Traction-Displacement Relationships}

Through the definition of a cohesive zone volume element (CZVE, Yamakov, et al. 2006) - an atomistic analog to a continuum CZM element - the results from MD simulations may be recast to obtain averaged continuum traction-displacement relationships that represent the cohesive interactions along some characteristic length of material. In Figure 7, stress and opening profiles are extracted along the crack growing in the system prestressed at 4.25 GPa hydrostatic load and exhibiting the deformation shown in Figure 6d. The insert in Figure 7 shows the schematic diagram of the slicing of the system volume in the MD simulation and defining the representative regions for extracting the parameters for the CZM elements suitable for use in a continuum simulation.

In Figure 7, a curve fit of $r^{-1 / 2}$ is shown as a close approximation to the $\sigma_{y y}^{s}(x)$ values extracted from the MD for the $+x$ crack tip and are consistent with the predominantly brittle fracture seen in this direction. In contrast, the plastic deformation processes, including twinning and dislocation emission, that dominate near the $-x$ direction crack tip, have a pronounced effect on the stress distribution. The $\sigma_{y y}^{s}(x)$ values extracted from the MD for the $-x$ crack tip are nearly constant with position, consistent with a strong plastic contribution. This asymmetric behavior is well explained by the Rice criterion (Rice, 1992) for cleavage vs. dislocation blunting transition at the crack tip. 


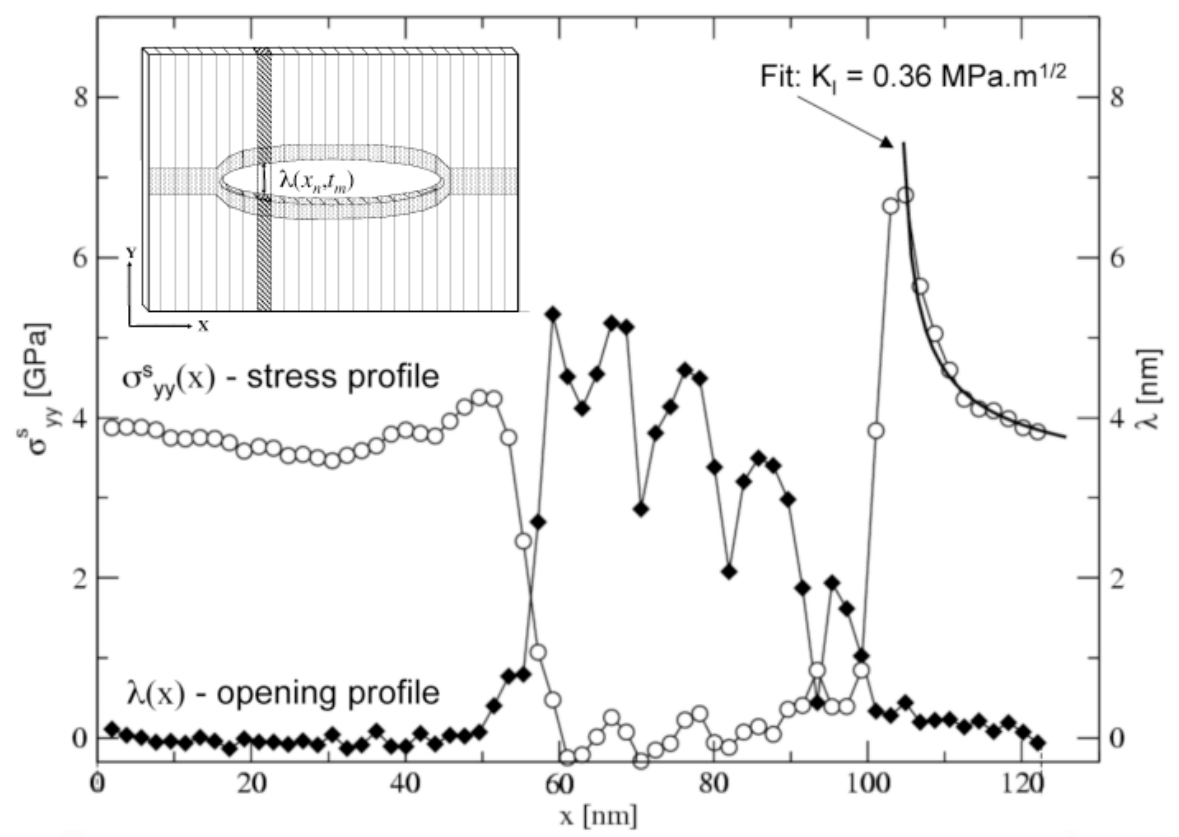

Figure 7. Stress and opening profiles extracted along the crack growing in a system prestressed at $4.25 \mathrm{GPa}$ hydrostatic load.

These differences in behavior suggest that two separate cohesive laws are needed to describe brittle and ductile crack propagation along one GB. The $\sigma_{y y}^{s}(x)$ and $\lambda(x)$ profiles in Figure 7 are for a single instant of time of the crack propagation. If the entire simulation of the interface debonding is divided into $N_{t}$ equal intervals of time $t_{q}$ $\left(q \in\left[0, N_{t}\right]\right)$, many such profiles can be taken of many CZVEs placed along the GB. When plotted in a $\sigma_{y y}^{s}$ vs. $\lambda$ plot, each $\left(\sigma_{y y}^{s}\left(x_{p}, t_{q}\right), \lambda\left(x_{p}, t_{q}\right)\right)$ couple represents a point $\sigma_{y y}^{s}\left(\lambda\left(x_{p}, t_{q}\right)\right)$. After sorting these data points in order of ascending $\lambda$, so that $\lambda_{i}<\lambda_{i+1}$, and taking a moving average (or a consecutive mean):

$$
\tau\left(\lambda_{i}\right)=\frac{1}{(2 M+1)} \sum_{j=-M}^{M} \sigma_{y y}^{s}\left(\lambda_{i+j}\right)
$$

in which the results are averaged over $M$ points backward and $M$ points forward from $\lambda_{i}$, a construction of a statistically representative traction-displacement function $\tau(\lambda)$ can be made. The calculated values for $\tau(\lambda)$ for the brittle and ductile crack tips are shown in Figure 8. Complete details of the modeling, fracture mechanics and extraction of the CZM response are given in Yamakov et al. (2006).

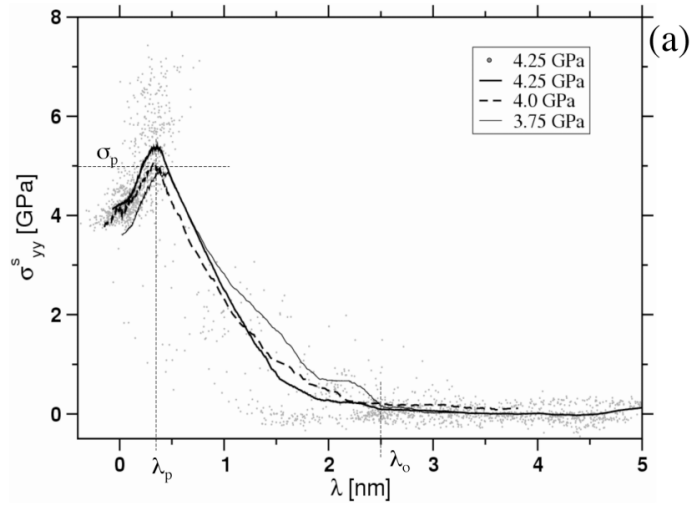

a) Brittle fracture CZM

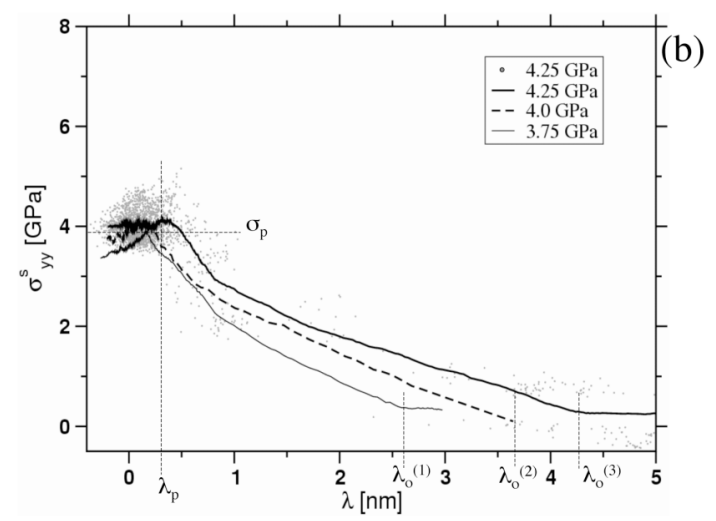

b) Ductile fracture CZM

Figure 8. Brittle and ductile traction-displacement relationships from MD simulation. 


\section{Parameterized Approach to Multiscale Modeling}

Although numerous forms of cohesive zone model (CZM) have been developed in the literature (Chandra et al., 2002), a general bilinear CZM is sufficient to approximate the traction-displacement response shown in Figure 8 for Mode I crack propagation along the $\Sigma 99$ GB configuration. By adjusting the parameters of the bilinear CZM, a range of peak tractions and opening displacements can be considered to represent variations in GB decohesion properties transitioning from the more brittle to the more ductile type of decohesion. These variations may arise from different GB misorientation angles, defining different types of GB structures (Wolf, 1990) as well as the presence of vacancies, interstitials, and other defects in the GB interface. The CZM then provides a numerically efficient, but qualitative, means of considering a broad range of GBs without atomistic modeling of all of the mechanisms and configurations that contribute to the variations in GB properties.

The traction-displacement relationships, along with corresponding works of separation (Eq. (1)), may take the CZM forms presented in Figure 9. The functional form of the CZMs may be varied to represent process zones that range from perfectly brittle to perfectly ductile. An intermediate model expressed as a general bilinear tractiondisplacement relationship is also shown in Figure 9 and represents the common situation in which a mixture of elastic and inelastic deformation mechanisms exists and characterizes crack propagation. Here, the parameters $\tau_{d t}^{o}$, $\tau_{m x}^{o}, \tau_{b r}^{o}$ represent the peak tractions of the perfectly brittle, intermediate, and perfectly ductile CZM models, respectively. Similarly, $\Delta_{d t}^{c}, \Delta_{m x}^{c}$, and $\Delta_{b r}^{c}$ represent the maximum displacements of the perfectly brittle, intermediate, and perfectly ductile CZM models, respectively.

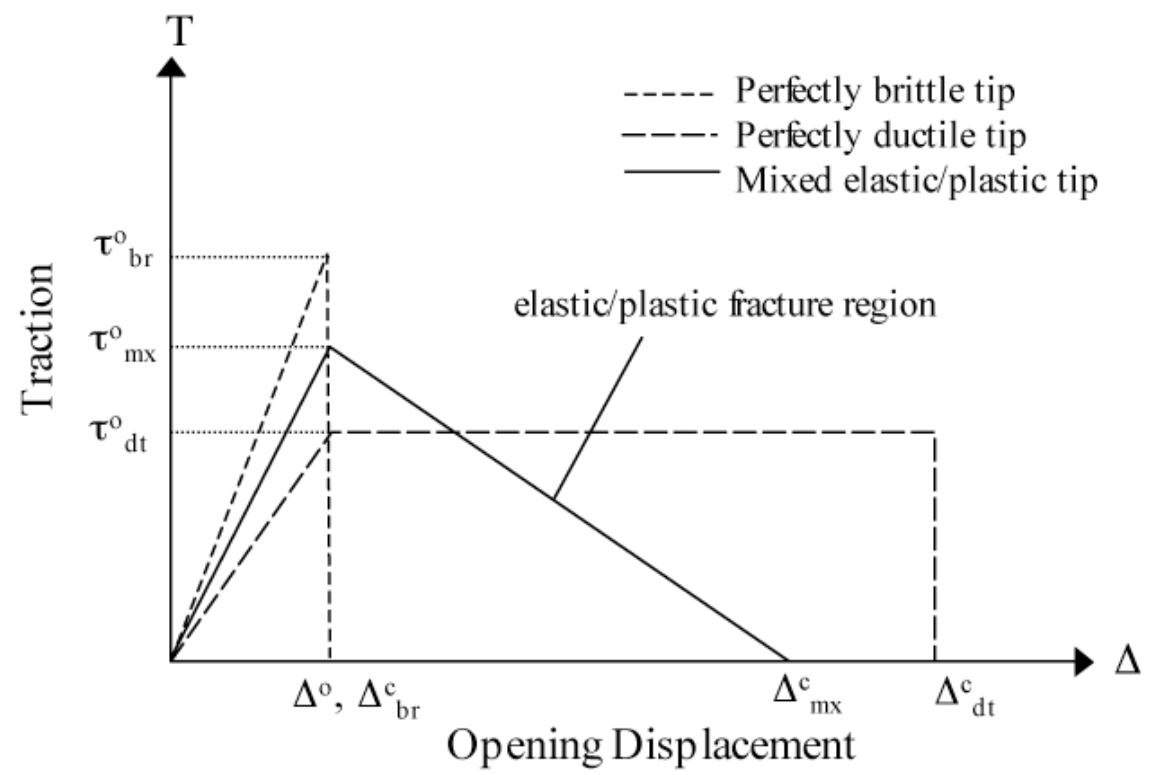

Figure 9. Possible traction-displacement relationships for perfectly brittle, perfectly ductile, and mixed elastic/plastic crack tip behavior.

\section{Microstructural Configurations}

Figure 10 shows the two configurations considered in the finite element analysis implementation of the parameterized cohesive zone models. The size of both configurations is chosen to be $l_{x}=l_{y}=380 \mathrm{~nm}$, so that the characteristic length of the cohesive zone element placed along the crack path is $l_{x} / 200=1.9 \mathrm{~nm}$, i.e., equal to the size of the CZVE used in the MD simulations (Yamakov et al, 2006). In both cases, 6-node triangular finite elements are used to represent the elastic continuum having the elastic properties of Aluminum ( $E=72 \mathrm{GPa}, v=0.32$ ). Figure 10a shows a simple bi-crystal configuration with a single interface between the two crystals and an initial crack of length $a_{o}=7.6 \mathrm{~nm}$. The model is loaded with a linearly increasing tensile displacement in the $y$-direction that increases from $U_{y}=0$ at $l_{x}=0$ to $U_{y}=6 \%$ strain at $l_{x}=380 \mathrm{~nm}$. Figure $10 \mathrm{~b}$ shows a more complex configuration consisting of a tessellated polycrystalline microstructure having many grain boundary interfaces but with no preexisting crack. The polycrystalline microstructure is simulated under a uniform tensile strain $U_{y}$ loaded incrementally to $22 \%$ strain. 


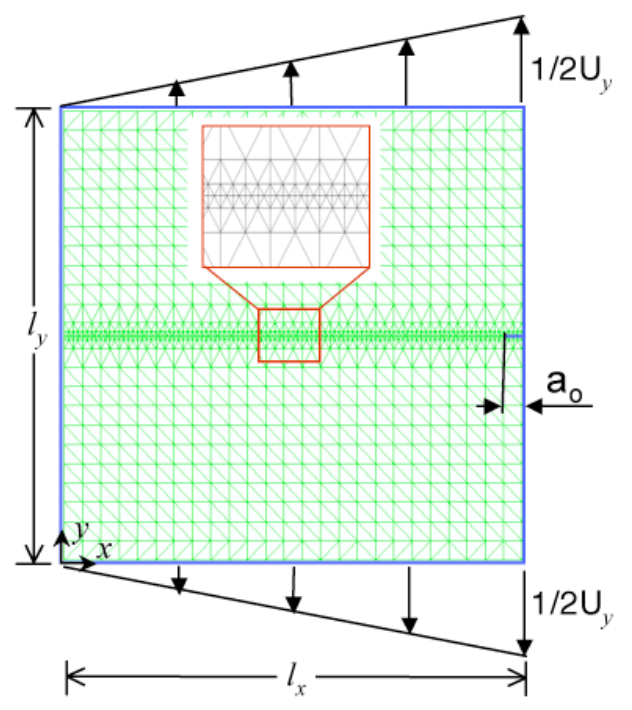

a) Edge cracked tension model

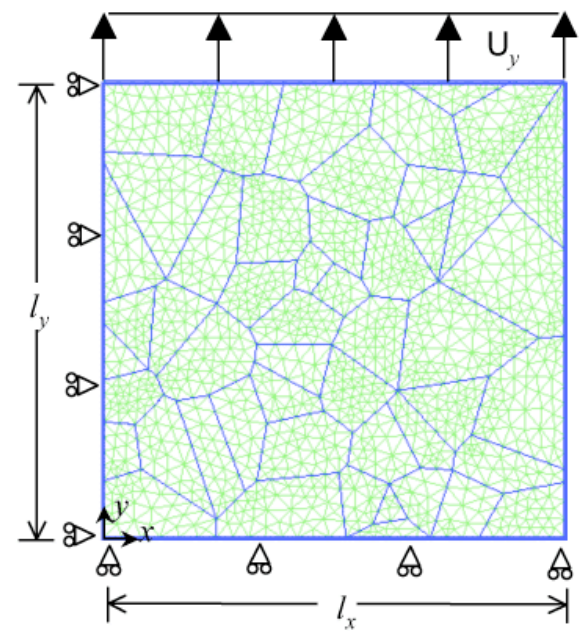

b) Random microstructure model

Figure 10. Cracked tension model for Mode I opening.

\section{Results for Parameterized CZM}

Parametric studies were performed to assess the effect of the relative amount of elastic and plastic deformation at the crack tip on the fracture characteristics of the configurations shown in Figure 10. This was achieved by varying the shape of the CZMs using the MD extracted cohesive zone curves shown in Figure 8 as a baseline. Two sets of parameterized CZMs were generated as shown in Figure 11. In the first set (Figure 11a), the effect of the relative amount of elastic and plastic deformation was varied, resulting in different values of the energy of crack face separation or toughness, $G$, while keeping $\tau_{m x}$ constant. In the second set (Figure $11 \mathrm{~b}$ ), the ratio of elastic to plastic deformation was varied by changing the maximum opening displacement, $\Delta_{m x}$, for CZMs having the same toughness. Keeping $G$ constant results in a varied maximum peak traction $\tau_{m x}$. In this way, the effect of the shape and the area of the CZM curve on the fracture properties of the interface can be demonstrated. In Figure 11, CZMs A1 and B1 correspond to the MD-generated results shown in Figure 8.

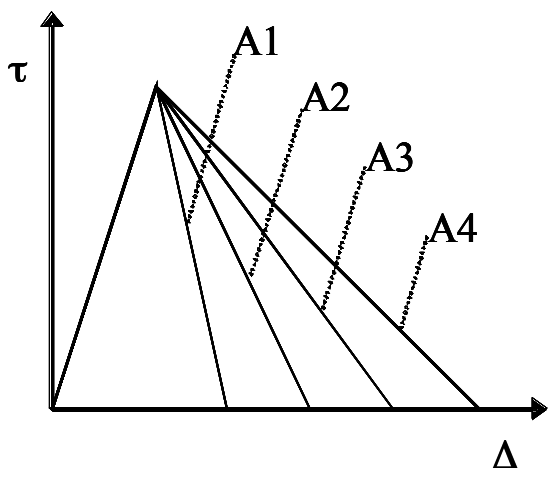

a) Series A: Equal peak traction

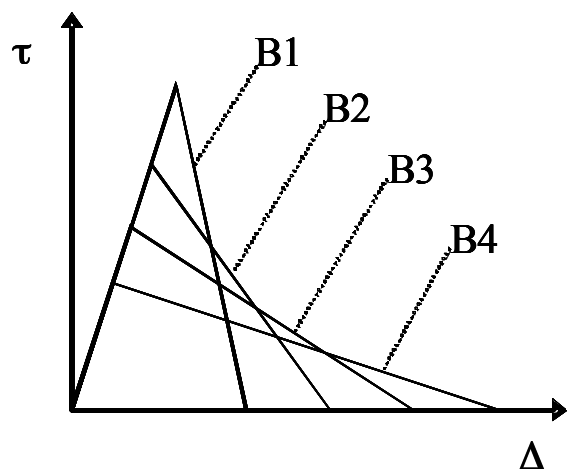

b) Series B: Equal toughness

Figure 11. Cohesive zone models used in two series of parametric studies.

The parameters used to define the models shown in Figure 11a and Figure 11b are shown in Tables 1a and 1b, respectively. 
Table 1a. CZM parameters used to simulate different relative elastic and plastic behavior at the crack tip. Fracture toughness is a function of the increase in plasticity. The peak traction is held constant at $\tau_{m x}^{c}=5.0 \mathrm{GPa}$.

\begin{tabular}{|c|c|c|}
\hline Cohesive Zone Model & $\Delta^{\mathrm{c}}{ }_{\mathrm{mx}}$ & $\mathrm{G}\left(\mathrm{J} / \mathrm{m}^{2}\right)$ \\
\hline $\mathrm{A} 1$ & 2.17 & 5.42 \\
\hline $\mathrm{A} 2$ & 2.89 & 7.22 \\
\hline $\mathrm{A} 3$ & 4.33 & 10.84 \\
\hline $\mathrm{A} 4$ & 6.50 & 16.25 \\
\hline
\end{tabular}

Table 1b. CZM parameters used to simulate different relative elastic and plastic behavior at the crack tip. Fracture toughness is held constant at $G=5.42 \mathrm{~J} / \mathrm{m}^{2}$.

\begin{tabular}{|c|c|c|c|}
\hline Cohesive Zone Model & $\tau_{\text {mx }}^{\mathrm{o}}(\mathrm{GPa})$ & $\Delta_{\mathrm{o}}(\mathrm{nm})$ & $\Delta_{\mathrm{mx}}^{\mathrm{c}}(\mathrm{nm})$ \\
\hline B1 & 5.00 & 1.07 & 2.17 \\
\hline B2 & 3.75 & 0.80 & 2.89 \\
\hline B3 & 2.50 & 0.53 & 4.33 \\
\hline B4 & 1.75 & 0.37 & 6.19 \\
\hline
\end{tabular}

Figures 12 and 13 show the results for the bicrystal configuration of Figure 10a. The length of the crack was determined in two ways (see the inset in Figure 12a). First, the length contributions of all CZM element integration points that exceeded the peak traction $\tau_{m x}$ were summed to give the position, $a_{1}$, of the front edge of the process zone at the crack tip where 'softening' starts. Second, the length contributions of only those CZMs that exhibited complete loss of stiffness were summed to give position, $a_{2}$, where the process zone ends and a free surface is formed. In this way, the fracture process zone is determined as the 'softening' region between $a_{1}$ and $a_{2}$.

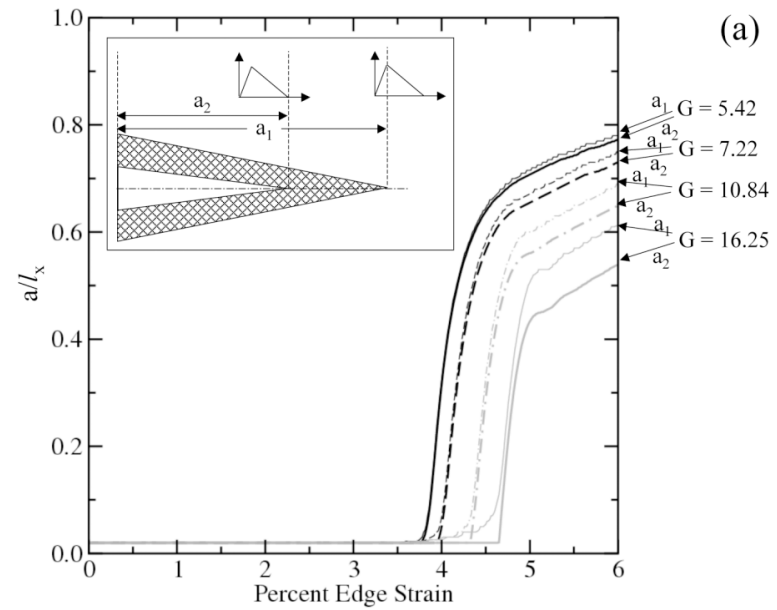

a) Measures $\mathrm{a}_{1}$ and $\mathrm{a}_{2}$ given separately

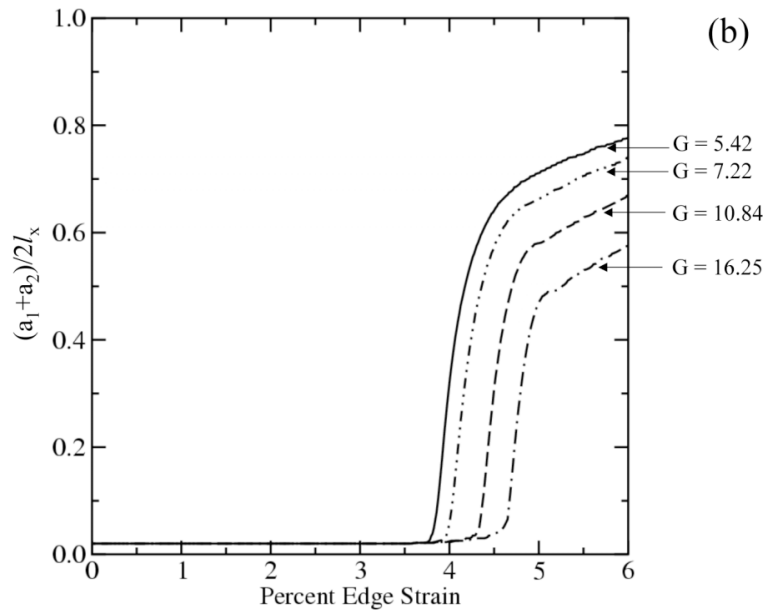

b) Mean crack length

Figure 12. Relative crack length vs. percent edge strain for CZM Series A.

Figure 12a shows the position $a_{1}$ and $a_{2}$ of the front and end of the crack process zone as a function of a linearly increasing far-field strain. Here, CZM Series A was considered. In all cases, the $a_{1}$ is greater than $a_{2}$ as it indicates the beginning of the processing zone. Note that the size of the process zone, estimated as the distance between the $a_{1}$ and $a_{2}$ lines for each $G$, increases with increasing $G$. In addition, the two sets of lines exhibit a systematic trend towards shorter crack lengths as the fracture toughness, $G$, increases. The shortening of the effective crack length, taken as the mean of the two positions $a_{1}$ and $a_{2}$ with increasing $G$, is observed in Figure 12b. In spite of the fact that 
the peak traction $\tau_{m x}$, corresponding to the onset of damage, was kept constant, the increase of $G$ leads to the crack opening at larger strains.

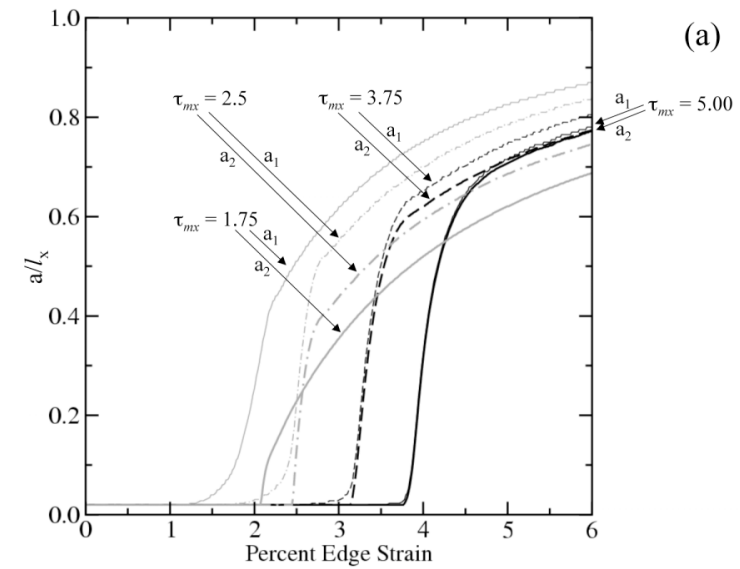

a) Measures $a_{1}$ and $a_{2}$ given separately

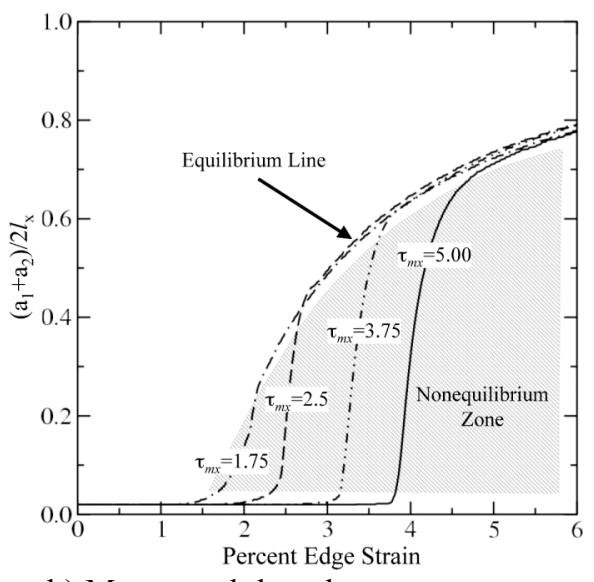

b) Mean crack length

Figure 13. Relative crack length vs. percent edge strain for CZM Series B.

Figure 13a presents the analogous results for CZM Series B. The same effect of increasing the process zone is found, but this time it is due to the increased size of the CZM plastic region by decreasing $\tau_{m x}$ while $G$ was kept constant. The effective crack length versus applied strain is shown in Figure 13b. Two different regions of crack growth can be seen. First, at the moment of opening, the crack is unstable and the mean crack length exhibits an instantaneous growth until it reaches a stable equilibrium. The zone of unstable growth is indicated by the shaded region in Figure 13b. Second, after reaching the equilibrium state, the equilibrium crack lengths for all $\tau_{m x}$ follow the common equilibrium master curve. This result shows that when $G$ is constant, the equilibrium crack length for a given strain remains the same and does not depend on the peak traction $\tau_{m x}$. Thus, the peak traction in the CZM affects only the initial strain of crack opening.

The results presented in Figure 14 demonstrate that the process zone in both CZM Series, A and B, systematically increases when the CZM parametric curve extends towards larger crack opening displacements, either through increasing of the fracture toughness at constant peak traction, or through decreasing of the peak traction while keeping the fracture toughness constant. Note that the decrease of $\tau_{m x}$ has a more pronounced effect on the process zone than increasing $G$ and indicates that lowering the traction barrier for debonding results in a larger increase of the processing zone in front of the crack tip compared to the case when $G$ is increased.

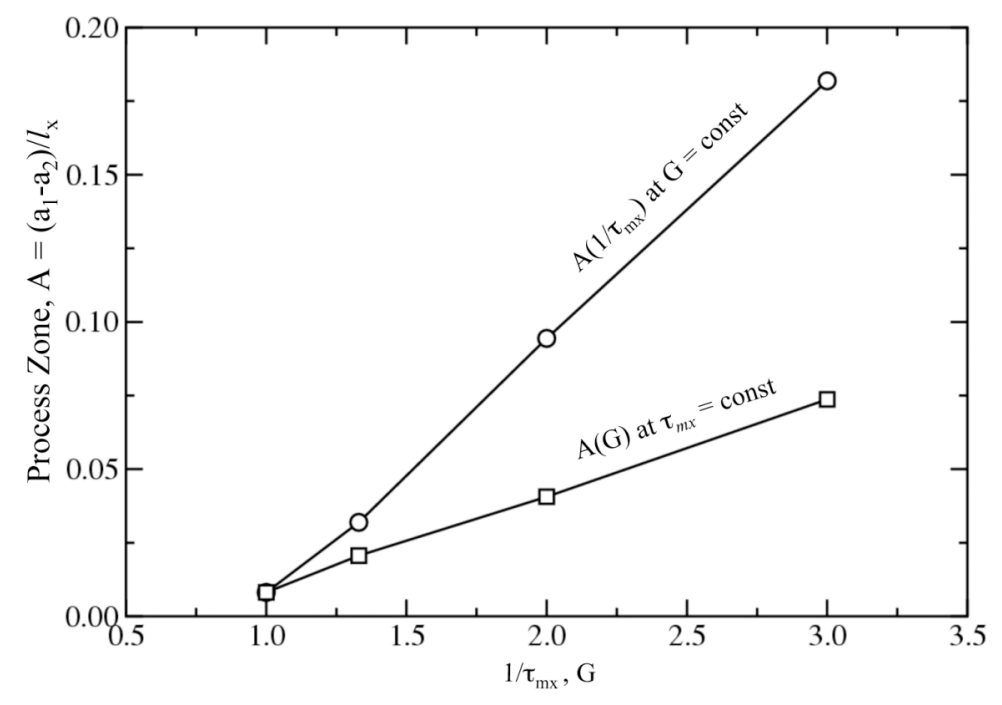

Figure 14. Process zone vs. $1 / \tau_{\mathrm{mx}}$ for Series B and Process zone vs. G for Series A. 
Although the MD results have only examined Mode I opening under very specific conditions for brittle and ductile crack tips along a $\Sigma 99$ grain boundary in pure aluminum, they provide a physical, albeit limited to nanometer scales, constitutive relation of decohesion that can lead to the construction of a database of CZMs for studying the failure of polycrystal microstructures. For example, by using the MD parameterized CZM traction-displacement relation in Figure 8a, a sequence of boundary opening states leading to the formation of a dominant microcrack in the configuration shown in Figure 10b can be illustrated as shown in Figure 15.

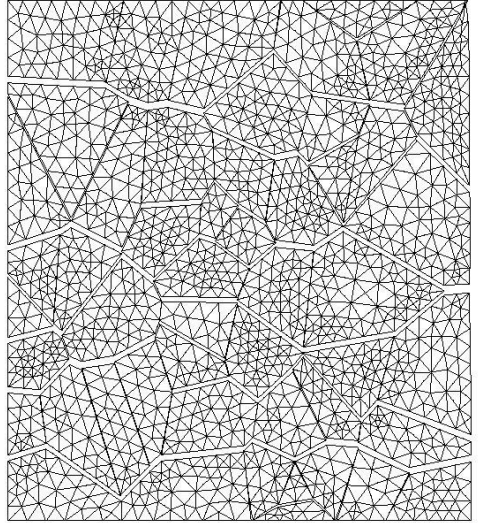

a) Initial opening of GBs, $\mathrm{U}_{\mathrm{y}}=16 \%$

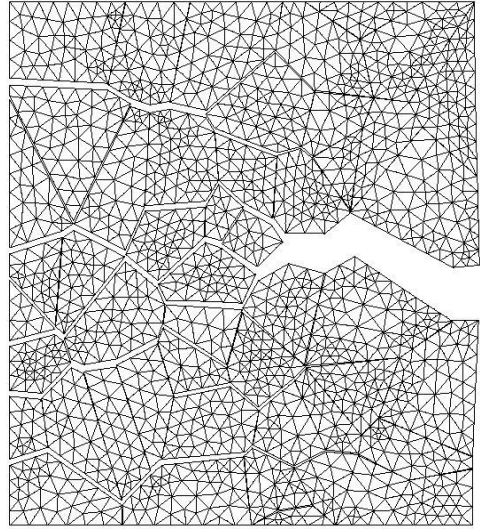

b) Local crack formation, $\mathrm{U}_{\mathrm{y}}=17 \%$

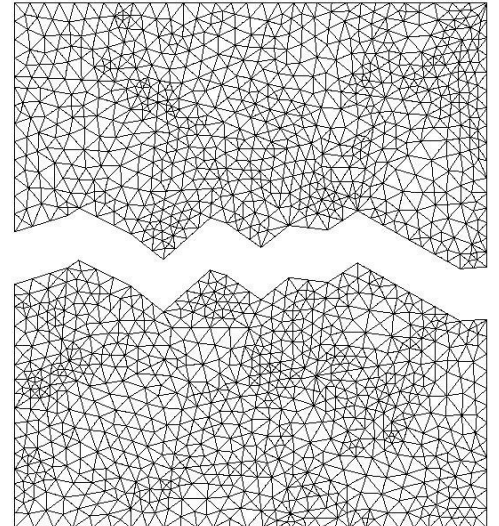

c) Dominant microcrack, $\mathrm{U}_{\mathrm{y}}=22 \%$

Figure 15. Process of dominant microcrack formation in a polycrystal model using cohesive zone models

An examination of the decohesion processes exhibited in the simulation presented in Figure 15 shows the initial opening of GBs under normal applied displacements. The CZM for Mode I opening obtained from MD analysis at this length scale has an initial GB stiffness that is of similar magnitude to the stiffness of the adjoining grains. Thus, these interfaces are shown to open at low levels of applied strain (Figure 15a). At higher applied strains, the energetics of GB opening dictate the location at which a dominant crack begins to form (Figure 15b). At a critical applied strain, the local coalescence of GB opening forms a dominant microcrack as shown in Figure 15c.

\section{Concluding Remarks}

The present methodology forms the basis for multiscale modeling of metallic fracture that is rooted at the atomistic level. Molecular dynamics simulations and a newly defined cohesive zone volume element were used to develop constitutive relationships describing interfacial deformation and fracture processes near a crack tip that were then recast within parameterized cohesive zone models and implemented as part of a finite element analysis. This methodology provides a physical, albeit limited to nanometer scales, constitutive relation of decohesion that can lead to the construction of a database of CZMs for studying the failure of polycrystal microstructures. In the present study the MD simulations considered nanoscale crack propagation along a common $\Sigma 99$ grain boundary in aluminum and yielded significantly different traction-displacement relationships for the crack growing in ductile and brittle directions along the grain boundary.

By adjusting the parameters of the bilinear CZM, a range of peak tractions and opening displacements were considered to represent variations in GB properties. These variations arise from the presence of vacancies, interstitials, and other defects in addition to the effect of the GB misorientation angle to the GB structure. The CZM then provides a numerically efficient means of considering a broad range of GBs incorporating mechanisms and configurations from the atomistic modeling that contribute to the variations in GB properties.

Using the atomistically-derived traction-displacement results as a basis, two different parameterizations for a CZM were considered where the relative amount of elastic and plastic deformation was varied either through varying the fracture toughness, while keeping the peak traction constant, or through varying the peak traction, while maintaining a constant fracture toughness. In both cases, the increase of plasticity results in an increase in the process zone size at the crack tip. Preserving the fracture toughness resulted in a constant equilibrium crack length at a fixed strain, independent of the peak traction. The peak traction affects the initial strain at which the crack opens. The CZM was then used to illustrate the formation of a dominant crack in a simple aluminum polycrystal. 


\section{Acknowledgments}

V. Yamakov and D. R. Phillips were sponsored through cooperative agreement NCC-1-02043 with the National Institute of Aerospace and contract NAS1-00135 with Lockheed Martin Space Operations, respectively. Helpful conversations with Dr. I. S. Raju are gratefully acknowledged.

\section{References}

${ }^{1}$ Benzeggagh, M.L., and Kenane, M., "Measurement of Mixed-Mode Delamination Fracture Toughnesss of Unidirectional Glass/Epoxy Composites With Mixed-Mode Bending Apparatus," Comp. Sci. and Tech., 49, 1996, pp. 439-439.

2 Camacho, G.T. and Ortiz, M., "Computational Modeling of Impact Damage in Brittle Materials," Int. J. Solids Struct., 33, 1996, pp. 2899-2938.

${ }^{3}$ Chandra, N., Li, H., Shet, C. and Ghonem, H., "Some Issues in the Application of Cohesive Zone Models for MetalCeramic Interfaces," Int. J. Solids Struct., 39, 2002, pp. 2827-2855.

${ }^{4}$ Clarke, A. S., and Jonsson, H., "Structural Changes Accompanying Densification of Random Hard-Sphere Packings," Phys. Rev. E, 47, 1993, pp. 3975-3984.

${ }^{5}$ Costanzo, F. and Allen, D. H., "A Continuum Thermodynamic Analysis of Cohesive Zone Models," Int. J. of Engrng. Scie., 33, 1995, pp. 2197-2219.

${ }^{6}$ Dávila, C. G., "Mixed-Mode Decohesion Elements for Analysis of Progressive Delamination," 42 AIAA/ASME/ASCE/AHS/ASC Structures, Structural Dynamics, and Materials Conference and Exhibit, Seattle, WA April, 1619 2001, article: AIAA-01-1486.

${ }^{7}$ Gall, K., Horstemeyer, M.F., Van Schilfgaarde, M., Baskes, M.I., "Atomistic Simulations on the Tensile Debonding of an Aluminum-Silicon Interface," J. Mech. Phys. Solids, 48, 2000, pp. 2183-2212.

${ }^{8}$ Glaessgen, E. H., Phillips, D. R., Yamakov, V., and Saether, E., "Multiscale Modeling for the Analysis of Grain-Scale Fracture within Aluminum Microstructures," Proceedings of the $46^{\text {th }}$ AIAA SDM Conference, April 18-21, 2005, Austin, TX, Paper No. AIAA-2005-1851.

9 Honeycutt, J. D., Andersen, H. C., "Molecular Dynamics Study of Melting and Freezing of Small Lennard-Jones Clusters," J. Phys. Chem., 91, 1987, pp. 4950-4963.

${ }^{10}$ Iesulauro, E., "Decohesion of Grain Boundaries in Statistical Representations of Aluminum Polycrystals," Cornell University Report 02-01, 2002.

${ }^{11}$ Klein, P. and Gao, H., "Crack Nucleation and Growth as Strain Localization in a Virtual-Bond Continuum," Engr. Fract. Mech, 61, 1998, pp. 21-48.

${ }^{12}$ Komanduri, R., Chandrasekaran, N., Raff, L.M., "Molecular Dynamics (MD) Simulation of Uniaxial Tension of Some Single-Crystal Cubic Metals at Nanolevel," Int. J. Mech. Sci., 43, pp. 2237-2260.

${ }^{13}$ Mishin, Y., et al., Phys. Rev. B, 59, 1999, p. 3393.

${ }^{14}$ Needleman, A., "An Analysis of Tensile Decohesion Along an Interface," J. Mech. and Phys. Solids, 38, 1990, pp. 289324.

${ }^{15}$ O'Rourke, J., Computational Geometry in C, $2^{\text {nd }}$ Edition, Cambridge University Press, 2001.

${ }^{16}$ Raynolds, J.E., Smith, J.R., Zhao, G.-L., Srolovitz, D.J., “Adhesion in NiAl-Cr from First Principles,” Phys. Rev. B, 53, 1996, pp. 13883-13890.

${ }^{17}$ Rice, J. R., "Dislocation Nucleation from a Crack Tip: An Analysis Based on the Peierls Concept," J. Mech. and Phys. Solids, 40, 1992, pp. 239-271.

${ }^{18}$ Spearot, D., Jacob, K.I., McDowell, D.L., "Non-Local Separation Constitutive Laws for Interfaces and Their Relation to Nanoscale Simulations," Mech. Mater., 36, 2004, pp. 825-847.

${ }^{19}$ Tadmor, E.B. and Hai, S., "A Peierls Criterion for the Onset of Deformation Twinning at a Crack Tip,” J. Mech. and Phys. Solids, 51, 2003, pp. 765-793.

${ }^{20}$ Turon, A., Camanho, P., Costa, J., and Davila, C., “An Interface Damage Model for the Simulation of Delamination Under Variable-Mode Ratio in Composite Materials,"NASA/TM-2004-213277, 2004.

${ }^{21}$ Tvergaard, V. and Hutchinson, J.W., "The Relation Between Crack Growth Resistance and Fracture Process Parameters in Elastic-Plastic Solids,” J. Mech. and Phys. Solids, 40, 1992, pp. 1377-1397.

${ }^{22}$ Wolf, D., 1990. "Correlation between structure, energy, and ideal cleavage fracture for symmetrical grain boundaries in fcc metals,” J. Mater. Res. 5, 1708-1730.

${ }^{23}$ Yamakov, V., Saether, E., Phillips, D.R., and Glaessgen, E.H., "Dynamic Instability in Intergranular Fracture,” Phys. Rev. Lett. 95, 2005, p. 015502.

${ }^{24}$ Yamakov, V., Saether, E., Phillips, D.R., and Glaessgen, E.H., "Molecular-Dynamics Simulation-Based Cohesive Zone Representation of Intergranular Fracture Processes in Aluminum," J. Mech. and Phys. Solids, 2006, (in press). 